\title{
TRANSLATION-EQUIVARIANT MATCHINGS OF COIN FLIPS ON $\mathbb{Z}^{d}$
}

\author{
TERRY SOO, * University of British Columbia
}

\begin{abstract}
Consider independent fair coin flips at each site of the lattice $\mathbb{Z}^{d}$. A translation-equivariant matching rule is a perfect matching of heads to tails that commutes with translations of $\mathbb{Z}^{d}$ and is given by a deterministic function of the coin flips. Let $Z_{\Phi}$ be the distance from the origin to its partner, under the translation-equivariant matching rule $\Phi$. Holroyd and Peres (2005) asked, what is the optimal tail behaviour of $Z_{\Phi}$ for translation-equivariant perfect matching rules? We prove that, for every $d \geq 2$, there exists a translationequivariant perfect matching rule $\Phi$ such that $\mathrm{E} Z_{\Phi}^{2 / 3-\varepsilon}<\infty$ for every $\varepsilon>0$.
\end{abstract}

Keywords: Matching; Bernoulli random field

2000 Mathematics Subject Classification: Primary 60G55; 60G60

Secondary $60 \mathrm{~K} 35$

\section{Introduction}

Consider the probability space $(\Omega, \mathcal{F}, \mathrm{P})$, where $\Omega=\{0,1\}^{\mathbb{Z}^{d}}, \mathcal{F}$ is the standard product $\sigma$-algebra of $\{0,1\}^{\mathbb{Z}^{d}}$, and $\mathrm{P}$ is the product measure on $\mathcal{F}$ with parameter $p=\frac{1}{2}$. We call elements of $\mathbb{Z}^{d}$ sites. For $\gamma \in \Omega$, a bijection $\phi: \mathbb{Z}^{d} \rightarrow \mathbb{Z}^{d}$ is a matching on $\gamma$ if every site $x$ with $\gamma(x)=1$ is mapped to a site $y$ with $\gamma(y)=0$, and vice versa, and if the composition $\phi \circ \phi$ is the identity mapping on $\mathbb{Z}^{d}$. For a site $z$, we define the translation $\theta^{z}$ on $\mathbb{Z}^{d}$ and $\Omega$ as follows: we set $\theta^{z} x:=x+z$ for all $x \in \mathbb{Z}^{d}$ and, for all $\gamma \in \Omega$, we set $\theta^{z} \gamma(x):=\gamma(x-z)$ for all $x \in \mathbb{Z}^{d}$. A measurable mapping $\Phi:\{0,1\}^{\mathbb{Z}^{d}} \times \mathbb{Z}^{d} \rightarrow \mathbb{Z}^{d}$ is a matching rule if $\Phi(\gamma, \cdot)$ is a matching on $\gamma$ for P-almost all $\gamma$. We say that $\Phi$ is translation equivariant if it commutes with translations; that is, $\Phi\left(\theta^{z} \gamma, \cdot\right)=\theta^{z} \Phi(\gamma, \cdot)$ for P-almost all $\gamma$.

Let $\|\cdot\|$ be the $l^{\infty}$ norm on $\mathbb{Z}^{d}$. We define $Z=Z_{\Phi}(\gamma):=\|\Phi(\gamma, \mathcal{O})\|$ to be the distance from the origin $\mathcal{O}=(0, \ldots, 0) \in \mathbb{Z}^{d}$ to its partner. We will construct a translation-equivariant matching rule $\Phi$ and obtain upper bounds on $\mathrm{P}(Z>r)$.

Theorem 1. For all $d \geq 1$, there exists a translation-equivariant matching rule $\Phi$ such that, for all $r>0$, we have

$$
\mathrm{P}(Z \phi>r) \leq c(\log r)^{4} r^{-\beta}
$$

for some $c=c(d)<\infty$, where $\beta=\beta(d)=2 /(1+4 / d)$.

Prior to this result, the best known decay appears to have been the following.

Theorem 2. ([8] and [10].) For $d \geq 1$, there exists a translation-equivariant matching rule $\Phi$ such that, for all $r>0$, we have $\mathrm{P}\left(Z_{\Phi}>r\right) \leq c r^{-1 / 2}$ for some $c=c(d)<\infty$.

Received 21 August 2008; revision received 13 August 2009.

* Postal address: Department of Mathematics, University of British Columbia, Vancouver BC, V6T 1Z2, Canada.

Email address: tsoo@math.ubc.ca 
Theorem 2 can be deduced from a simple construction due to Meshalkin [10]. Meshalkin's matching was originally used to construct isomorphisms of Bernoulli schemes [10]; it is the following construction. In $d=1$ we define a translation-equivariant matching rule inductively, by first matching a 0 to a 1 whenever a 0 is immediately to the left of a 1 , i.e.

$$
\text { ..0110 } \underline{01} 1111000 \underline{01} \ldots
$$

In the next stage we remove the matched pairs, and then follow the same procedure. It is straightforward to check that bounding $\mathrm{P}(Z>r)$ amounts to bounding $R=\inf \left\{m \geq 1: S_{m}=0\right\}$, where $S_{m}$ denotes a simple symmetric random walk.

We may deduce the $d \geq 2$ case of Theorem 2 from the following observation. By applying a translation-equivariant matching rule $\Phi_{d-1}$ on $\mathbb{Z}^{d-1}$ to each $(d-1)$-dimensional plane, given by $\{z\} \times \mathbb{Z}^{d-1}$ for each $z \in \mathbb{Z}$, we obtain a translation-equivariant matching rule $\Phi_{d}$ on $\mathbb{Z}^{d}=\mathbb{Z} \times \mathbb{Z}^{d-1}$, where $\mathrm{P}\left(Z_{\Phi_{d-1}}>r\right)=\mathrm{P}\left(Z_{\Phi_{d}}>r\right)$ for all $r>0$.

Theorem 1 provides faster decay than that provided by Theorem 2 for all $d>1$. After this paper was written, Timár [12] proved the following stronger result.

Theorem 3. ([12].) For any $d \geq 1$, there exists a translation-equivariant matching rule $\Phi$ such that, for all $r>0$, we have $\mathrm{P}\left(Z_{\Phi}>r\right) \leq c r^{-d / 2}$ for some $c=c(d)<\infty$.

Some of the methods of this paper also appear in [12]. New ideas are introduced in [12] and the methods of [12] are much more sophisticated.

For $d=1,2$, the bounds obtained in Theorem 3 are essentially best possible.

Theorem 4. ([6] and [8].) If $d=1,2$ then, for any translation-equivariant matching rule $\Phi$, we have $\mathrm{E} Z_{\Phi}^{d / 2}=\infty$.

Hence, for $d=1,2$, there does not exist a translation-equivariant matching rule $\Phi$ where $\mathrm{P}\left(Z_{\Phi}>r\right) \leq c r^{-\rho}$ for some constants $\rho>d / 2$ and $c=c(d)<\infty$.

Note that the result of Theorem 4 is only valid for $d=1,2$. In fact, for $d \geq 3$, Timár [12] showed that it is possible to find a translation-equivariant matching rule with even faster decay than that given by Theorem 3 .

Theorem 5. ([12].) For any $d \geq 3$ and any $\varepsilon>0$, there exists a translation-equivariant matching rule $\Phi$ such that, for all $r>0$, we have

$$
\mathrm{P}(Z>r) \leq C \exp \left(-c r^{d-2-\varepsilon}\right)
$$

for some constants $0<c, C<\infty$.

Variants of matching in continuum settings have also been studied; see [4], [5], [9], [11], and the references therein.

The proof of Theorem 1 will proceed in two steps. We will construct a translation-equivariant matching and then determine bounds for it. To construct a translation-equivariant matching, we will define, in a measurable translation-equivariant way, a sequence $P_{n}$ of successively coarser partitions of $\mathbb{Z}^{d}$. Following [7], we call $P_{n}$ a clumping rule. The members of $P_{n}$ are called clumps or $n$-clumps, and we call the clumping rule locally finite if all the clumps are bounded. A component of a clumping rule is a limit of some increasing (with respect to set inclusion) sequence of clumps. A clumping rule is connected if it has only one component. Adapting the construction in [7] we will construct a locally finite connected clumping rule. From a locally finite connected clumping rule, it is easy to obtain a translation-equivariant matching rule $\Phi$; 
this is because a translation-equivariant matching rule can be defined by first matching as many sites as possible within each 1-clump and then iteratively matching as many unmatched sites as possible in each $n$-clump for $n=2,3, \ldots$ We will obtain, with the central limit theorem and a version of the mass transport principle, a preliminary result which implies that, for $d \geq 3$ and all $\varepsilon>0$, we have $\mathrm{P}\left(Z_{\Phi}>r\right) \leq c r^{-3 / 5-\varepsilon}$ for some constant $c=c(d, \varepsilon)<\infty$. The preliminary result will not provide faster decay than that given by Theorem 2 in the cases $d=1,2$. Upon closer analysis of the geometry of the clumps, we will show that clumps that are long and thin happen with small probability; this analysis is the basis of the proof of Theorem 1.

The outline of the paper is as follows. In Section 2 we discuss clumping rules and matchings from clumping rules. In Section 3 we outline the construction of a clumping rule and collect some useful bounds. In Section 4 we introduce a version of the mass transport principle that will be useful in the proof of Theorem 1. In Section 5 we prove Theorem 1. We conclude the paper with some related open problems.

\section{Clumps}

Let $\mathcal{P}_{F}\left(\mathbb{Z}^{d}\right)$ denote all finite subsets of $\mathbb{Z}^{d}$. For $A \subset \mathbb{Z}^{d}$, define translations of $A$ via $\theta^{z} A:=\left\{\theta^{z} x: x \in A\right\}$. Formally, a locally finite connected clumping rule is a measurable mapping $\mathcal{C}:\{0,1\}^{\mathbb{Z}^{d}} \times \mathbb{N} \times \mathbb{Z}^{d} \rightarrow \mathcal{P}_{F}\left(\mathbb{Z}^{d}\right)$ with the following properties. For all $\gamma \in\{0,1\}^{\mathbb{Z}^{d}}$, all $n \in \mathbb{N}$, and all $x, y, z \in \mathbb{Z}^{d}$, we have

(i) $x \in \mathcal{C}(\gamma, n, x)$,

(ii) $\mathcal{C}(\gamma, n, x) \cap \mathcal{C}(\gamma, n, y) \neq \varnothing$ implies that $\mathcal{C}(\gamma, n, x)=\mathcal{C}(\gamma, n, y)$,

(iii) $\mathcal{C}(\gamma, n, x) \subset \mathcal{C}(\gamma, n+1, x)$,

(iv) $\mathcal{C}\left(\theta^{z} \gamma, n, \theta^{z} x\right)=\theta^{z} \mathcal{C}(\gamma, n, x)$,

(v) $\bigcup_{n} \mathcal{C}(\gamma, n, \mathcal{O})=\mathbb{Z}^{d}$.

Properties (i) and (ii) assure us that, for each $n \in \mathbb{N}$, the map $\mathcal{C}(\cdot, n, \cdot)$ is a partition. Property (iii) makes the partition successively coarser, (iv) is translation equivariance, and (v) is connectedness.

Proposition 1. There exists a locally finite connected clumping rule almost surely.

The proof of Proposition 1 will be given in the next section.

\subsection{Matchings from clumpings}

From a locally finite connected clumping rule we can construct a translation-equivariant matching rule in a countable number of stages. In the first stage, within each of the 1-clumps we match every possible site. Given that the $(n-1)$ th stage is completed, within each of the $n$-clumps we match every site we can, ignoring the sites that were previously matched. In order to ensure that the resulting matching is translation-equivariant, use, for example, a lexicographic ordering on $\mathbb{Z}^{d}$, to determine the maximal partial matching on the clumps. Ergodicity, connectedness, and the fact that $p=\frac{1}{2}$ give us that every site will be matched at some stage. Note that for our purposes we do not need to make this argument as we obtain upper bounds on $\mathrm{P}(Z>r)$, which easily imply that $\mathrm{P}(Z>r) \rightarrow 0$ as $r \rightarrow \infty$ (see Theorem 1 or Proposition 4, below).

In the next section we construct an explicit locally finite connected clumping rule $\mathcal{C}$. 


\section{Seeds, cutters, and blobs}

Our construction of the clumping rule $\mathcal{C}$ is adapted from [7]. In [7] and [13] clumpings are used to obtain factor graphs of point processes. See also [2] for background.

\subsection{Basic set-up}

Let $\|\cdot\|$ denote the $l^{\infty}$ norm on $\mathbb{Z}^{d}$. Let $S(x, r):=\left\{y \in \mathbb{R}^{d}:\|x-y\| \leq r\right\}$. Thus, $S(x, r)$ is the cube of side length $2 r$ centered at $x$. We also write $S(\mathcal{O}, r)=S(r)$. We let $\left\{e_{m}\right\}_{m=1}^{d}$ be the standard unit basis vectors in $\mathbb{R}^{d}$.

For each $k \in \mathbb{N}$, we say that a site $x \in \mathbb{Z}^{d}$ is a $k$-seed if $\gamma(x)=1$ and $\gamma(y)=0$ for all $y \in$ $\left\{x+n e_{1}: 1 \leq n \leq k-1\right\}$. Whenever $x$ is a $k$-seed we call the set $\left\{x+n e_{1}: 0 \leq n \leq k-1\right\}$ its shell. For example, a 4 -shell has the form 1000 . Note that the probability of a $k$-seed occurring at a particular point is exactly $2^{-k}$. Two seeds are said to be overlapping if their shells intersect. Note that two seeds $x$ and $y$ overlap if and only if $x=y$. This property will be useful later (see Section 5.2). We define

$$
r_{k}=\left(2^{k} k^{2}\right)^{1 / d}+\frac{1}{2}
$$

The reason for the choice of $r_{k}$ will be evident shortly. Define the vector

$$
s_{k}:=\left\lfloor 100 r_{k}\right\rfloor e_{1} .
$$

A $k$-cutter is a subset of $\mathbb{R}^{d}$ of the form $\left\{y \in \mathbb{R}^{d}:\|y-x\|=r_{k}\right\}$, where $x-s_{k}$ is a $k$-seed. We introduce a shift $s_{k}$ for technical reasons which will surface later. We define $W_{k} \subset \mathbb{R}^{d}$ to be the union of all the $k$-cutters. Note that we have chosen $r_{k}$ so that $r_{k} \notin \mathbb{N}$. Thus, we have $W_{k} \cap \mathbb{Z}^{d}=\varnothing$ for all $k \in \mathbb{N}$. A $k$-blob is a connected component of $\mathbb{R}^{d} \backslash \bigcup_{j>k} W_{j}$. Hence, the sequence of $k$-blobs defines a successively coarser partition of $\mathbb{R}^{d}$ (ignoring the elements of $\bigcup_{k} W_{k}$.) The $k$-blobs induce a clumping rule $\mathcal{C}$ when we intersect the $k$-blobs with $\mathbb{Z}^{d}$. Note the technical distinction between blobs and clumps.

It is obvious that the induced clumping rule $\mathcal{C}$ is translation equivariant; it remains to show that it is locally finite and connected. It suffices to show that all the blobs are bounded and that, for every $x \in \mathbb{R}^{d}$, there is a $k$-blob that contains both $x$ and the origin.

\subsection{Estimates}

In this section we obtain some estimates that will show that the clumping rule $\mathcal{C}$ defined in the previous section is indeed locally finite and connected. The following events will be important in our analysis. Let

$$
E_{k}(x):=\{x \text { is enclosed by some } k \text {-cutter }\}
$$

that is, $E_{k}(x)$ occurs if and only if, for some site $x_{0}, x_{0}-s_{k}$ is a $k$-seed and

$$
x \in\left\{y \in \mathbb{Z}^{d}:\left\|y-x_{0}\right\| \leq r_{k}\right\} .
$$

Also, let $E_{k}=E_{k}(\mathcal{O})$. Let

$$
U_{k}(s):=\{S(s) \text { intersects some } k \text {-cutter }\}
$$

that is, $U_{k}(s)$ occurs if and only if, for some site $x_{0}, x_{0}-s_{k}$ is a $k$-seed and

$$
\left\{y \in \mathbb{R}^{d}:\left\|y-x_{0}\right\|=r_{k}\right\} \cap S(s) \neq \varnothing .
$$


Also, let

$$
C_{k}(s):=\bigcup_{j \geq k} U_{k}(s) .
$$

From an analysis of these events we will deduce that the clumping rule $\mathcal{C}$ is both locally finite and connected. Moreover, we will see that the tail behavior of $Z_{\Phi}$ (where $\Phi$ is a translationequivariant matching rule obtained from the clumping rule $\mathcal{C}$ ) also depends on these events.

Lemma 1. (Enclosure bounds.) For all $k>c_{1}$, for some $c_{1}=c_{1}(d)<\infty$, we have

$$
\mathrm{P}\left(E_{k}^{\mathrm{c}}\right) \leq \mathrm{e}^{-k} \text {. }
$$

Proof. Note that

$$
E_{k}=\left\{S\left(-s_{k}, r_{k}-1\right) \text { contains some } k \text {-seed }\right\} .
$$

Let $p_{k}$ be the maximum possible number of $k$-seeds inside $S\left(-s_{k}, r_{k}-1\right)$. Recall that no two (distinct) $k$-seeds overlap and that the probability that a $k$-seed occurs at a particular point is $2^{-k}$. Hence, $\mathrm{P}\left(E_{k}\right) \geq 1-\left(1-2^{-k}\right)^{p_{k}} \geq 1-\mathrm{e}^{-2^{-k} p_{k}}$. By our choice of $r_{k}$ in (1) and since $\left(r_{k}^{d} / k\right) \leq p_{k} \leq\left\lceil 2 r_{k} / k\right\rceil\left(2 r_{k}\right)^{d-1}$ for all $k \geq c_{1}$, for some $c_{1}=c_{1}(d)<\infty$, we have $\mathrm{P}\left(E_{k}\right) \geq 1-\mathrm{e}^{-k}$.

Corollary 1. All k-blobs are bounded almost surely.

Proof. It suffices to show that all $k$-blobs that contain $\mathcal{O}$ are bounded. By Lemma 1 we have $\mathrm{P}\left(E_{k}\right) \rightarrow 1$ as $k \rightarrow \infty$, so that $E_{k}$ occurs for infinitely many $k$ almost surely. Hence, all blobs which contain $\mathcal{O}$ are bounded.

Lemma 2. (Cutter bounds.) For all $k \geq 1$ and all $s>0$, we have $\mathrm{P}\left(C_{k}(s)\right) \leq c_{3} s\left(k^{2} / r_{k}\right)$ for some $c_{3}=c_{3}(d)>0$.

Proof. Observe that

$$
U_{k}(s)=\left\{S\left(-s_{k}, r_{k}+s\right) \backslash S\left(-s_{k}, r_{k}-s\right) \text { contains some } k \text {-seed }\right\} .
$$

Clearly, $\mathrm{P}\left(U_{k}(s)\right) \leq N_{k}(s) 2^{-k}$, where $N_{k}(s)$ is the number of lattice points in $S\left(-s_{k}, r_{k}+s\right) \backslash$ $S\left(-s_{k}, r_{k}-s\right)$. We have

$$
N_{k}(s)=\left|S\left(r_{k}+s\right)\right|-\left|S\left(r_{k}-s\right)\right| \leq c_{2} r_{k}^{d-1} s
$$

for some $c_{2}=c_{2}(d)>0$. So we obtain $\mathrm{P}\left(U_{k}(s)\right) \leq c_{2} s r_{k}^{d-1} 2^{-k}$. Thus, recalling our choice of $r_{k}$ in (1), we have

$$
\mathrm{P}\left(C_{k}(s)\right) \leq \sum_{j \geq k} \mathrm{P}\left(U_{j}(s)\right) \leq c_{2} s \sum_{j \geq k} 2^{-k} r_{k}^{d-1} \leq c_{3} s\left(\frac{k^{2}}{r_{k}}\right) .
$$

Corollary 2. The clumping rule $\mathcal{C}$ is connected almost surely.

Proof. Let $s>0$. By the Borel-Cantelli lemma and (6), we know that $U_{k}(s)$ occurs infinitely often with probability 0 . Thus, any point within distance $s$ of $\mathcal{O}$ will eventually share a blob with it.

Proof of Proposition 1. The proof follows by applying Corollaries 1 and 2.

Now we obtain a translation-equivariant matching rule $\Phi$ from our locally finite connected clumping rule $\mathcal{C}$, via the procedure outlined in Section 2 . We will use Lemmas 1 and 2 and the central limit theorem to obtain bounds on $Z_{\Phi}$. 


\section{Mass transport}

We will require a version of the mass transport principle in order to facilitate calculations. See [1] and [3] for background. Our main application of the mass transport principle will be to prove a modified version of Lemma 3, below, which states that each site has an equal chance of not being matched within its $k$-clump. Similar ideas also appear in [9].

Let $\mathcal{C}$ be the clumping rule defined in Section 3, and let $\Phi$ be the corresponding translationequivariant matching rule obtained from $\mathcal{C}$. We say that a site is $k$-bad if it is not matched in its $k$-clump. Let $L_{k}(x)$ be the $k$-clump containing the site $x$, and let $L_{k}(\mathcal{O})=L_{k}$ be the $k$-clump containing the origin. Let $\#\left[L_{k}\right]$ be the cardinality of $L_{k}$. Consider the sum $\zeta:=\sum_{x \in L_{k}}(2 \gamma(x)-1)$, so that $|\zeta|$ is the number of $k$-bad sites in $L_{k}$.

Lemma 3. For all $k \geq 1$, the probability that the origin is $k$-bad is exactly

$$
\mathrm{E}\left(\frac{1}{\#\left[L_{k}\right]}\left|\sum_{x \in L_{k}}(2 \gamma(x)-1)\right|\right) .
$$

We define a mass transport to be a nonnegative measurable function

$$
T: \mathbb{Z}^{d} \times \mathbb{Z}^{d} \times\{0,1\}^{\mathbb{Z}^{d}} \rightarrow \mathbb{R}
$$

which is translation equivariant; that is, for all $x, y \in \mathbb{Z}^{d}$, all $\gamma \in\{0,1\}^{\mathbb{Z}^{d}}$, and all translations $\theta$ of $\mathbb{Z}^{d}$, we have $T(\theta x, \theta y, \theta \gamma)=T(x, y, \gamma)$. For $A, B \subset \mathbb{Z}^{d}$, we let $T(A, B, \gamma):=$ $\sum_{x \in A, y \in B} T(x, y, \gamma)$. We think of $T(A, B, \gamma)$ as the mass transferred from $A$ to $B$ under $\gamma \in \Omega$. We will use the following version of the mass transport principle.

Lemma 4. (Mass transport principle.) For any mass transport, $T: \mathbb{Z}^{d} \times \mathbb{Z}^{d} \times\{0,1\}^{\mathbb{Z}^{d}} \rightarrow \mathbb{R}$, we have $\mathrm{E} T\left(\mathcal{O}, \mathbb{Z}^{d}, \cdot\right)=\mathrm{E} T\left(\mathbb{Z}^{d}, \mathcal{O}, \cdot\right)$.

Proof. We have

$$
\begin{aligned}
\operatorname{E} T\left(\mathcal{O}, \mathbb{Z}^{d}, \cdot\right) & =\sum_{y \in \mathbb{Z}^{d}} \int T(\mathcal{O}, y, \gamma) \mathrm{dP}(\gamma) \\
& =\sum_{y \in \mathbb{Z}^{d}} \int T\left(-y, \mathcal{O}, \theta^{-y} \gamma\right) \mathrm{dP}(\gamma) \\
& =\sum_{y \in \mathbb{Z}^{d}} \int T(-y, \mathcal{O}, \gamma) \mathrm{dP}(\gamma) \\
& =\operatorname{E} T\left(\mathbb{Z}^{d}, \mathcal{O}, \cdot\right) .
\end{aligned}
$$

The first and last equalities follow from Fubini's theorem. The second equality follows from the translation equivariance of $T$ and the third equality follows from the translation invariance of $P$.

To illustrate the versatility of the mass transport principle (Lemma 4), we prove the following (unsurprising) fact.

Proposition 2. Let $\mathcal{F}$ be the standard product $\sigma$-algebra of $\{0,1\}^{\mathbb{Z}^{d}}$, and let $\mathrm{P}_{p}$ be the product measure on $\mathcal{F}$ with parameter $p$. If $p \neq \frac{1}{2}$ then there does not exists a translation-equivariant matching rule. 
Proof. Let $\Phi$ be a translation-equivariant matching rule. Consider the mass transport $M$ defined as follows. Let $x$ be a site, and let $\gamma \in \Omega$. If $\gamma(x)=1$ then $M(x, x, \gamma)=1$; that is, $x$ sends one unit of mass to itself. Otherwise, $M(x, y, \gamma)=1$, where $y$ is a site with $\Phi(x, \gamma)=y$ and $\gamma(y)=1$; that is, $x$ sends out a unit of mass to the site $y$ that it is matched to under $\Phi(\cdot, \gamma)$. Since $\Phi$ is translation equivariant, this defines a mass transport $\mathrm{P}_{p}$-almost surely. Let $\mathrm{E}_{p}$ be the expected value operator with respect to the measure $\mathrm{P}_{p}$. Now, since every site sends out exactly one unit of mass, we have $\mathrm{E}_{p} M\left(\mathcal{O}, \mathbb{Z}^{d}, \cdot\right)=1$. Also, by considering the cases $\gamma(\mathcal{O})=1$ or $\gamma(\mathcal{O})=0$, we also have $\mathrm{E}_{p} M\left(\mathbb{Z}^{d}, \mathcal{O}, \cdot\right)=2 p$. Hence, we have, by the mass transport principle, $p=\frac{1}{2}$.

Proof of Lemma 3. For each $k \geq 1$, we define a mass transport $T_{k}$ by saying that if a site $x$ is $k$-bad then it sends out one unit of mass uniformly to every site in its $k$-clump $L_{k}(x)$, while $x$ sends out no mass if the site is not bad. To be precise,

$$
T_{k}(x, y, \gamma):=\left(\frac{1}{\#\left[L_{k}(x)\right]}\right) \mathbf{1}_{[x \text { is } k \text {-bad }]}(\gamma) \mathbf{1}_{\left[y \in L_{k}(x)\right]}(\gamma) .
$$

It is easy to see that

$$
\mathrm{E} T_{k}\left(\mathbb{Z}^{d}, \mathcal{O}, \cdot\right)=\mathrm{E}\left(\frac{1}{\#\left[L_{k}\right]} \sum_{x \in L_{k}} \mathbf{1}_{[x \text { is } k \text {-bad }]}\right) .
$$

On the other hand, we have $\mathrm{E} T_{k}\left(\mathcal{O}, \mathbb{Z}^{d}, \cdot\right)=\mathrm{P}\{\mathcal{O}$ is $k$-bad $\}$. Thus, an application of the mass transport principle completes the proof.

Now we are in a position to obtain bounds on $\mathrm{P}(Z>r)$. We will see that the mass transport principle with information about the size of $L_{k}$ and its diameter gives us an estimate with an application of the central limit theorem.

\section{Proof of Theorem 1}

\subsection{First estimates}

Let $\Phi$ be the translation-equivariant matching rule we obtain from the clumping rule $\mathcal{C}$ defined in Section 3. Recall that $Z=Z_{\Phi}$ is the distance from the origin to its partner under $\Phi$. We will obtain bounds on $\mathrm{P}(Z>r)$ by choosing a sequence of events $D_{k}$ and a $K=K(r)$ so that $\{Z>r\} \cap D_{K} \subset\{\mathcal{O}$ is $K$-bad $\}$. The events $D_{k}$ will be chosen in a such way that we can obtain upper bounds on $\mathrm{P}\{\mathcal{O}$ is $K$-bad $\}$ and $\mathrm{P}\left(D_{K}^{\mathrm{c}}\right)$.

Let $\alpha \in(0,1)$. In fact, we will end up choosing $\alpha=\alpha(d)=1 /(1+d / 4)$. Recall that the events $E_{k}$ and $C_{k}(s)$ were defined earlier in Section 3.2; see (2) and (3). Let $B_{k}$ be the $k$-blob containing the origin. The following relations describe the geometry of $B_{k}$, when $E_{k}$ or $C_{k}\left(r^{\alpha}\right)$ occur. We have

$$
E_{k} \subset\left\{\text { there exists } x \text { so that } B_{k} \subset S\left(x, r_{k}\right) \subset S\left(2 r_{k}\right)\right\}
$$

and

$$
C_{k}\left(r^{\alpha}\right)^{\mathrm{c}} \subset\left\{S\left(r^{\alpha}\right) \subset B_{k}\right\} .
$$

We consider the following decomposition:

$$
\{Z>r\}=\left(\left(E_{k} \cap C_{k}\left(r^{\alpha}\right)^{\mathrm{c}}\right) \cap\{Z>r\}\right) \cup\left(\left(E_{k}^{\mathrm{c}} \cup C_{k}\left(r^{\alpha}\right)\right) \cap\{Z>r\}\right) .
$$

See Figure 1 for a realization of the event $E_{k} \cap C_{k}\left(r^{\alpha}\right)^{\mathrm{c}}$. 


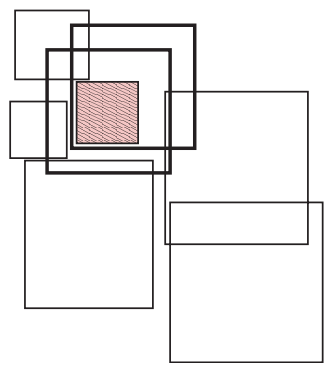

FIGURE 1: An illustration of the event $E_{k} \cap C_{k}\left(r^{\alpha}\right)^{\mathrm{c}}$. The thick cutters represent $k$-cutters enclosing the origin. This corresponds to the event $E_{k}$. The shaded region represents the no cutter zone of radius $r^{\alpha}$ about the origin. This corresponds to the event $C_{k}\left(r^{\alpha}\right)^{\mathrm{c}}$.

The role of parameter $\alpha$ can be explained heuristically as follows. If the parameter $\alpha$ is small then $C_{k}\left(r^{\alpha}\right)^{\mathrm{c}}$ occurs with high probability, but then $B_{k}$ could possibly be very small. If $\alpha$ is close to 1 then $B_{k}$ would almost contain a cube of length $2 r$, but then $C_{k}\left(r^{\alpha}\right)^{\mathrm{c}}$ occurs with low probability. We will choose $\alpha$ to optimize over these alternatives.

Let $K=K(r)$ be defined to be the unique integer $K$ such that

$$
2 r_{K}<r_{K+1}<r \leq r_{K+2} \text {. }
$$

Note that, for some $c_{4}=c_{4}(d)>0$, we have, for all $k \geq c_{4}, r_{k}=\left(2^{k} k^{2}\right)^{1 / d}+\frac{1}{2} \leq\left(\mathrm{e}^{k} / 2^{-k}\right)^{1 / d}$. Hence, applying (1) with (10) for all sufficiently large $r$ we have

$$
\left(\frac{\log 2}{1+\log 2}\right)(K+1) \leq \frac{d \log r}{1+\log 2} \leq K+2 .
$$

Proposition 3. (Decay of the first term in (9) via the central limit theorem.) For all $r>0$ and the unique integer $K=K(r)$ such that $r_{K+1}<r \leq r_{K+2}$, we have

$$
\mathrm{P}\left(\left(E_{K} \cap C_{K}\left(r^{\alpha}\right)^{\mathrm{c}}\right) \cap\{Z>r\}\right) \leq \frac{c_{5}}{\left(r^{\alpha}\right)^{d / 2}}
$$

for some $c_{5}=c_{5}(d)>0$.

Remark. Note that the decay in Proposition 3 is the decay that appears in Theorem 3, if we set $\alpha=1$.

Before we begin the proof of Proposition 3, we collect some easy, but important, observations. By (7) and (10), we have

$$
\left(E_{K} \cap C_{K}\left(r^{\alpha}\right)^{\mathrm{c}}\right) \cap\{Z>r\} \subset\{\mathcal{O} \text { is } K \text {-bad }\} .
$$

So from (8) we have

$$
\left(\left(E_{K} \cap C_{K}\left(r^{\alpha}\right)^{\mathrm{c}}\right) \cap\{Z>r\}\right) \subset\left(\{\mathcal{O} \text { is } K \text {-bad }\} \cap E_{K} \cap\left\{\#\left[L_{K}\right] \geq r^{\alpha d}\right\}\right) .
$$

Recall that $L_{k}$ is the $k$-clump containing $\mathcal{O}$. To analyze the right-hand side of (12), we will use the following version of Lemma 3. 
Lemma 5. For all $k \geq 1$, we have

$$
\begin{aligned}
& \mathrm{P}\left(\{\mathcal{O} \text { is } k-b a d\} \cap E_{k} \cap\left\{\#\left[L_{k} \geq r^{\alpha d}\right]\right\}\right) \\
& \quad=\mathrm{E}\left(\frac{1}{\#\left[L_{k}\right]}\left|\sum_{x \in L_{k}}(2 \gamma(x)-1)\right| ; E_{k} \cap\left\{\#\left[L_{k}\right] \geq r^{\alpha d}\right\}\right) .
\end{aligned}
$$

Proof. We use the mass transport principle. Recall $T_{k}$ as defined in the proof of Lemma 3:

$$
T_{k}(x, y, \gamma):=\left(\frac{1}{\#\left[L_{k}(x)\right]}\right) \mathbf{1}_{[x \text { is } k \text {-bad }]}(\gamma) \mathbf{1}_{\left[y \in L_{k}(x)\right]}(\gamma) .
$$

We define another mass transport, namely

$$
\hat{T}_{k}(x, y, \gamma):=T_{k}(x, y, \gamma) \mathbf{1}_{\left[E_{k}(x) \cap\left\{\#\left[L_{k}(x)\right] \geq r^{\alpha d}\right\}\right]}(\gamma) .
$$

Note that, on the event $\left\{y \in L_{k}(x)\right\}$, the event $E_{k}(x)$ occurs if and only if the event $E_{k}(y)$ occurs and $\#\left[L_{k}(x)\right]=\#\left[L_{k}(y)\right]$. Hence, we obtain

$$
\begin{aligned}
\mathrm{E} \hat{T}_{k}\left(\mathbb{Z}^{d}, \mathcal{O}, \cdot\right) & =\mathrm{E} \sum_{y \in \mathbb{Z}^{d}} T_{k}(y, \mathcal{O}, \cdot) \mathbf{1}_{\left[E_{k}(y) \cap\left\{\#\left[L_{k}(y)\right] \geq r^{\alpha d}\right\}\right]} \\
& =\mathrm{E} \sum_{y \in \mathbb{Z}^{d}} T_{k}(y, \mathcal{O}, \cdot) \mathbf{1}_{\left[E_{k} \cap\left\{\#\left[L_{k}\right] \geq r^{\alpha d}\right\}\right]} \\
& =\mathrm{E}\left(\frac{1}{\#\left[L_{k}\right]}\left|\sum_{x \in L_{k}}(2 \gamma(x)-1)\right| ; E_{k} \cap\left\{\#\left[L_{k}\right] \geq r^{\alpha d}\right\}\right) .
\end{aligned}
$$

On the other hand, we have

$$
\mathrm{E} \hat{T}_{k}\left(\mathcal{O}, \mathbb{Z}^{d}, \cdot\right)=\mathrm{P}\left(\{\mathcal{O} \text { is } k-\operatorname{bad}\} \cap E_{k} \cap\left\{\#\left[L_{k}\right] \geq r^{\alpha d}\right\}\right) .
$$

Thus, an application of the mass transport principle (Lemma 4) completes the proof.

Next we will use the central limit theorem to estimate the right-hand side of (13), but first we need to verify that we have the necessary independence. For $k \geq 1$, consider the events

$$
H_{k}\left(x_{1}, x_{2}, \ldots, x_{n}\right):=\left\{\left\{x_{1}, \ldots, x_{n}\right\}=L_{k} \cap S\left(2 r_{k}\right)\right\},
$$

where $x_{i} \in \mathbb{Z}^{d}$ and $\left\|x_{i}\right\| \leq 2 r_{k}$. Let $g_{k}:=\sigma\left(\gamma(x): x \in S\left(2 r_{k}\right)\right)$. The following lemma is behind why the (large) shift $s_{k}=\left\lfloor 100 r_{k}\right\rfloor e_{1}$, along the axis $e_{1}$, appears in the definition of the $k$-cutters.

Lemma 6. For all $k \geq 1$ and all $\left\|x_{i}\right\| \leq 2 r_{k}$, the $\sigma$-field $g_{k}$ is independent of

$$
\sigma\left(H_{k}\left(x_{1}, \ldots, x_{n}\right), E_{k}\right) .
$$

Proof. Consider a site $y$ with $\|y\|<s_{k} / 3$. The event $\left\{y \in L_{k}\right\}$ is determined by whether there exist $j$-seeds, with $j \geq k$, to give rise to $j$-cutters that can separate $y$ from $\mathcal{O}$. However, such $j$-seeds (and their shells) are at least at distance $s_{k} / 2$ from the origin. So, $\{\gamma(x):\|x\|<$ $\left.s_{k} / 3\right\}$ is independent of $H_{k}\left(x_{1}, x_{2}, \ldots, x_{n}\right)$ for all $x_{i} \in \mathbb{Z}^{d}$ such that $\left\|x_{i}\right\| \leq 2 r_{k}$. Also, recall that $E_{k}$ from (4) is determined by $\gamma(x)$, where $\|x\| \geq s_{k} / 2$. 
Now the proof of Proposition 3 amounts to a simple calculation, whose result we record in the next lemma.

Lemma 7. For all $k \geq 1$, we have

$$
\mathrm{P}\left(\{\mathcal{O} \text { is } k-b a d\} \cap E_{k} \cap\left\{\#\left[L_{k}\right] \geq r^{\alpha d}\right\}\right) \leq \frac{c_{5}}{\left(r^{\alpha}\right)^{d / 2}}
$$

for some $c_{5}>0$.

Proof of Proposition 3. From (12) and Lemma 7, Proposition 3 follows immediately.

Proof of Lemma 7. Let $k \geq 1$, and recall that by (7) we know that on the event $E_{k}$ we have $L_{k} \subset S\left(2 r_{k}\right)$. Fix $x_{1}, \ldots, x_{n} \in S\left(2 r_{k}\right)$, and let $H_{k}=H_{k}\left(x_{1}, \ldots, x_{n}\right)$. We will now compute

$$
A:=\mathrm{E}\left(\frac{1}{\#\left[L_{k}\right]}\left|\sum_{x \in L_{k}}(2 \gamma(x)-1)\right| ; E_{k} \cap H_{k}\left(x_{1}, \ldots, x_{n}\right)\right),
$$

by conditioning on $g_{k}$. Let $S_{n}=\sum_{i=1}^{n}\left(2 \gamma\left(x_{i}\right)-1\right)$. Consider the following calculation:

$$
\begin{aligned}
A & =\mathrm{E}\left(n^{-1}\left|\sum_{i=1}^{n}\left(2 \gamma\left(x_{i}\right)-1\right)\right| \mathbf{1}_{\left[E_{k} \cap H_{k}\right]}\right) \\
& =\mathrm{E}\left(\mathrm{E}\left(n^{-1}\left|S_{n}\right| \mathbf{1}_{\left[E_{k} \cap H_{n}\right]} \mid g_{k}\right)\right) \\
& =\mathrm{E}\left(\frac{1}{n}\left|S_{n}\right| \mathrm{E}\left(\mathbf{1}_{\left[E_{k} \cap H_{k}\right]} \mid g_{k}\right)\right) \\
& =\mathrm{E}\left(\frac{1}{n}\left|S_{n}\right|\right) \mathrm{E}\left(\mathbf{1}_{\left[E_{k} \cap H_{k}\right]}\right) \\
& \leq \frac{c_{5}}{\sqrt{n}} \mathrm{P}\left(E_{k} \cap H_{k}\right)
\end{aligned}
$$

for some $c_{5}>0$. Equality (14) is obtained by conditioning on the $\sigma$-field $g$. Equality (15) comes from the fact that the $\gamma\left(x_{i}\right)$ are all $g$ measurable. By Lemma 6 we know that $g_{k}$ and $\sigma\left(H_{k}, E_{k}\right)$ are independent, thus establishing equality (16). Inequality (17) is obtained by applying the central limit theorem.

By summing over all possible $H_{k}\left(x_{1}, \ldots, x_{n}\right)$ we obtain

$$
\mathrm{E}\left(\frac{1}{\#\left[L_{k}\right]}\left|\sum_{x \in L_{k}}(2 \gamma(x)-1)\right| ; E_{k} \cap\left\{\#\left[L_{k}\right]=n\right\}\right) \leq \frac{c_{5}}{\sqrt{n}} \mathrm{P}\left\{\#\left[L_{k}\right]=n\right\} .
$$

Furthermore, by summing over all $n \geq r^{\alpha d}$ we see that

$$
\mathrm{E}\left(\frac{1}{\#\left[L_{k}\right]}\left|\sum_{x \in L_{k}}(2 \gamma(x)-1)\right| ; E_{k} \cap\left\{\#\left[L_{k}\right] \geq r^{\alpha d}\right\}\right) \leq \frac{c_{5}}{\left(r^{\alpha}\right)^{d / 2}} .
$$

Thus, an application of Lemma 5 completes the proof.

Now we turn our attention to the second term in (9): $\left(E_{k}^{\mathrm{c}} \cup C_{k}\left(r^{\alpha}\right)\right) \cap\{Z>r\}$. We will bound this term in two different ways. As a first step, let us just throw away the term $\{Z>r\}$, since this will allow us to obtain a novel result for the case $d \geq 3$ without much more additional effort. 
Lemma 8. (Decay of the second term in (9): first bound.) For all $r>0$ and the unique integer $K=K(r)$ such that $r_{K+1}<r \leq r_{K+2}$, we have

$$
\mathrm{P}\left(E_{K}^{\mathrm{c}} \cup C_{K}\left(r^{\alpha}\right)\right) \leq c_{6} r^{\alpha-1}(\log r)^{2}
$$

for some $c_{6}=c_{6}(d)>0$.

Proof. Recall that, from Lemma 1 and Lemma 2, we already have bounds for the events appearing in this term. From (11) we see that

$$
\mathrm{P}\left(E_{K}^{c}\right) \leq c_{7} r^{-d /(1+\log 2)}
$$

for some $c_{7}=c_{7}(d)>0$. Note that $d /(1+\log 2)>d / 2$. On the other hand, applying (11) to Lemma 2 we obtain

$$
\mathrm{P}\left(C_{K}\left(r^{\alpha}\right)\right) \leq c_{8} r^{\alpha-1}(\log r)^{2}
$$

for some $c_{8}=c_{8}(d)>0$.

Proposition 4. (Easy preliminary result.) For all $d \geq 1$, there exists a translation-equivariant matching rule $\Phi$ such that $Z=Z_{\Phi}(\gamma)=\|\Phi(\gamma, \mathcal{O})\|$ has the tail behavior $\mathrm{P}(Z>r) \leq$ $c_{9} r^{-\beta^{\prime}}(\log r)^{2}$, where $c_{9}=c_{9}(d)>0$ and $\beta^{\prime}=\beta^{\prime}(d)=1 /(1+2 / d)$.

Proof. We can see, from (9), Proposition 3, and Lemma 8, that

$$
\mathrm{P}(Z>r) \leq c_{5} r^{-\alpha d / 2}+c_{6} r^{\alpha-1}(\log r)^{2} .
$$

Hence, we are led to minimize the quantity $\max (-\alpha d / 2, \alpha-1)$. So we choose (for the purposes of this proposition) $\alpha=\alpha(d)=1 /(1+d / 2)$.

Proposition 4 gives, for $d=2$, a decay of order $(\log r)^{2} / r^{1 / 2}$. For $d=2$, Theorem 2 still provides a better result, but, for $d \geq 3$, Proposition 4 provides faster decay than Theorem 2 .

\subsection{Long and thin blobs}

With a closer analysis of the second term in (9) we will prove the following.

Proposition 5. (Decay of the second term in (9): closer analysis.) For all $r>0$ and the unique integer $K=K(r)$ such that $r_{K+1}<r \leq r_{K+2}$, we have

$$
\mathrm{P}\left(\left(E_{K}^{c} \cup C_{K}\left(r^{\alpha}\right)\right) \cap\{Z>r\}\right) \leq c_{10} r^{-\alpha d / 2}+c_{11} r^{2(\alpha-1)}(\log r)^{4}
$$

for some $c_{10}=c_{10}(d)>0$ and $c_{11}=c_{11}(d)>0$.

Proposition 5 together with Proposition 3 yields a proof of Theorem 1.

Proof of Theorem 1. From the previous results, (9), Proposition 3, and Proposition 5, we have

$$
\mathrm{P}\left(Z_{\Phi}>r\right) \leq c_{5} r^{-\alpha d / 2}+c_{10} r^{-\alpha d / 2}+c_{11} r^{2(\alpha-1)}(\log r)^{4} .
$$

Hence, we are led to minimize the quantity $\max (-\alpha d / 2,2(\alpha-1))$. It is easy to verify that we should take $\alpha(d)=1 /(1+d / 4)$. Let $\beta(d):=d \alpha(d) / 2=2 /(1+4 / d)$. Thus, we obtain

$$
\mathrm{P}\left(Z_{\Phi}>r\right) \leq c(\log r)^{4} r^{-\beta},
$$

where $c=c(d)<\infty$ and $\beta=\beta(d)=2 /(1+4 / d)$. 


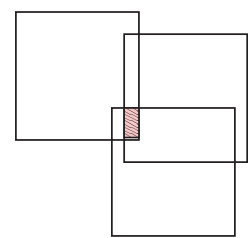

Figure 2: The shaded region represents the $k$-blob containing the origin. Note that on the event $C_{k}^{2}\left(r^{\alpha}\right)$ the $k$-blob can be quite small. For this reason, it seems we will not be able to do any better by including $\{Z>r\}$.

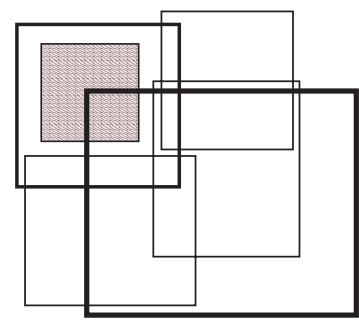

FiguRe 3: An illustration of the event $C_{k}^{1}\left(r^{\alpha}\right) \cap E_{k}$. The shaded region represents the restricted cutter zone of radius $r^{\alpha}$ about the origin. The very thick cutter represents the unique $j \geq k$ that intersects $S\left(r^{\alpha}\right)$. This corresponds to the event $C_{k}^{1}\left(r^{\alpha}\right)$. The other thick cutter represents a $k$-cutter enclosing the origin. This corresponds to the event $E_{k}$.

We will now work towards a proof of Proposition 5. We will need to examine the geometry of the blobs a bit closer to prove Proposition 5. Again, in light of (18) we do not need to worry about the event $E_{k}^{\mathrm{c}}$. Let us consider the decomposition,

$$
C_{k}\left(r^{\alpha}\right)=\left(E_{k}^{\mathrm{c}} \cap C_{k}\left(r^{\alpha}\right)\right) \cup\left(E_{k} \cap C_{k}\left(r^{\alpha}\right)\right) .
$$

The second term puts us in a position akin to the situation of Proposition 3, since we can control the diameter of the $k$-blob containing the origin. We now examine two situations. One where the $k$-blob containing $\mathcal{O}$ is possibly very small (see Lemma 9, below, and Figure 2) and another where there are enough points inside the $k$-blob to make good use of the central limit theorem (see Lemma 11, below, and Figure 3).

Let $j \geq k$. Consider again $j$-seeds on the sets

$$
A_{j}=A_{j}\left(r^{\alpha}\right):=S\left(-s_{j}, r_{j}+r^{\alpha}\right) \backslash S\left(-s_{j}, r_{j}-r^{\alpha}\right) .
$$

Observe that seeds on two levels will not overlap; that is, a seed in $A_{j}$ will not overlap with a seed in $A_{m}$ for $j \neq m$. Also, recall that, by our definition of $k$-seeds, no two (distinct) $k$-seeds will overlap. Since $C_{k}\left(r^{\alpha}\right)$ is the event that, for some $j \geq k$, the set $A_{j}$ contains a $j$-seed, we will further split up this event. Define

$$
\begin{aligned}
C_{k}^{1}\left(r^{\alpha}\right):= & \text { for all } j \geq k, \text { the set } A_{j} \text { contains at most one } j \text {-seed and there is a } \\
& \text { unique } \left.j \geq k \text { such that } A_{j} \text { contains a } j \text {-seed }\right\}, \\
C_{k}^{2}\left(r^{\alpha}\right):= & C_{k}\left(r^{\alpha}\right) \backslash C_{k}^{1}\left(r^{\alpha}\right) .
\end{aligned}
$$


We will throw away the term $\{Z>r\}$ when we bound $\mathrm{P}\left(C_{k}^{2}\left(r^{\alpha}\right) \cap E_{k} \cap\{Z>r\}\right)$, but we will keep it when we bound $\mathrm{P}\left(C_{k}^{1}\left(r^{\alpha}\right) \cap E_{k} \cap\{Z>r\}\right)$.

Lemma 9. For all $r>0$ and the unique integer $K=K(r)$ such that $r_{K+1}<r \leq r_{K+2}$, we have $\mathrm{P}\left(C_{K}^{2}\left(r^{\alpha}\right)\right) \leq c_{13}\left(r^{\alpha-1}(\log r)^{2}\right)^{2}$ for some $c_{13}=c_{13}(d)>0$.

Proof. For all $j \geq 1$, let $U_{j}=\left\{A_{j}\right.$ contains a $j$-seed $\}$. Thus, from (5) we have $U_{j}=U_{j}\left(r^{\alpha}\right)$. Since, for all $j \geq 1$, no two (distinct) $j$-seeds overlap, we have

$$
\mathrm{P}\left\{A_{j} \text { contains more than one } j \text {-seed }\right\} \leq \mathrm{P}\left(U_{j}\right)^{2} .
$$

Similarly, since seeds in $A_{j}$ and $A_{m}$ do not overlap for $j \neq m$, we have $\mathrm{P}\left(U_{j} \cap U_{m}\right)=$ $\mathrm{P}\left(U_{j}\right) \mathrm{P}\left(U_{m}\right)$ for all $j \neq m$. Since

$$
C_{k}^{2}\left(r^{\alpha}\right) \subset\left(\bigcup_{j>m \geq k} U_{j} \cap U_{m}\right) \cup\left(\bigcup_{j \geq m}\left\{A_{j} \text { contains more than one } j \text {-seed }\right\}\right),
$$

we have

$$
\mathrm{P}\left(C_{k}^{2}\left(r^{\alpha}\right)\right) \leq \sum_{j \geq k} \mathrm{P}\left(U_{j}\right) \sum_{m \geq k} \mathrm{P}\left(U_{m}\right) .
$$

By (6) and (11), it is easy to see that $\mathrm{P}\left(C_{K}^{2}\left(r^{\alpha}\right)\right) \leq c_{13}\left(r^{\alpha-1}(\log r)^{2}\right)^{2}$ for some $c_{13}=$ $c_{13}(d)>0$. Thus, we have an improved term $r^{2(\alpha-1)}$. For a realization of the event $C_{k}^{2}\left(r^{\alpha}\right)$, see Figure 2.

We now turn our attention to the event $C_{k}^{1}\left(r^{\alpha}\right)$.

Lemma 10. For all $r>0$ and the unique integer $K=K(r)$ such that $r_{K+1}<r \leq r_{K+2}$, we have $C_{K}^{1}\left(r^{\alpha}\right) \subset\left\{\#\left[L_{K}\right] \geq c_{14} r^{\alpha d}\right\}$ for some constant $0<c_{14}=c_{14}(d)<\infty$.

Proof. On the event $C_{K}^{1}\left(r^{\alpha}\right)$, there is exactly one $j$-cutter that has the property that it intersects $S\left(r^{\alpha}\right)$ and $j \geq K$; call this unique cutter $C$. Observe that if the cutter $C$ was removed, the blob containing the origin would contain all of $S\left(r^{\alpha}\right)$. Note that $C$ has side length at least $2 r_{K}$. It is easy to see that there is a constant $c_{15}<\infty$ independent of $k$, so that $c_{15} r_{k} \geq r_{k+2}$. Thus, $c_{15} r_{K} \geq r$. Therefore, the blob containing the origin must contain a $d$-cube with side length $r^{\alpha} / c_{15}$.

Lemma 11. For all $r>0$ and the unique integer $K=K(r)$ such that $r_{K+1}<r \leq r_{K+2}$, we have $\mathrm{P}\left(C_{K}^{1}\left(r^{\alpha}\right) \cap E_{K} \cap\{Z>r\}\right) \leq c_{12} /\left(r^{\alpha}\right)^{d / 2}$ for some $c_{12}=c_{12}(d)>0$.

Proof. Again, from (7) and (10), we have $C_{K}^{1}\left(r^{\alpha}\right) \cap E_{K} \cap\{Z>r\} \subset\{\mathcal{O}$ is $K$-bad $\}$. So, by Lemma 10, it suffices to show that, for all $k \geq 1$, we have

$$
\mathrm{P}\left(\{\mathcal{O} \text { is } k \text {-bad }\} \cap E_{k} \cap\left\{\#\left[L_{k}\right] \geq c_{14} r^{\alpha d}\right\}\right) \leq \frac{c_{12}}{\left(r^{\alpha}\right)^{d / 2}}
$$

for some $c_{12}=c_{12}(d)>0$. Equation (21) follows from Lemma 7 .

Proof of Proposition 5. Using (19) and (20), we have

$$
\mathrm{P}\left(\left(E_{K}^{c} \cup C_{K}\left(r^{\alpha}\right)\right) \cap\{Z>r\}\right) \leq \mathrm{P}\left(E_{K}^{c}\right)+\mathrm{P}\left(C_{K}^{2}\left(r^{\alpha}\right)\right)+\mathrm{P}\left(\left(C_{K}^{1}\left(r^{\alpha}\right)\right) \cap E_{K} \cap\{Z>r\}\right) .
$$

From (18) and Lemmas 9 and 11, we obtain

$$
\mathrm{P}\left(\left(E_{K}^{c} \cup C_{K}\left(r^{\alpha}\right)\right) \cap\{Z>r\}\right) \leq c_{7} r^{-d /(1+\log 2)}+c_{13}\left(r^{\alpha-1}(\log r)^{2}\right)^{2}+\frac{c_{12}}{\left(r^{\alpha}\right)^{d / 2}} .
$$




\section{Open problems}

1. What is the optimal tail behavior for translation-equivariant matchings on $\mathbb{Z}^{d}$ in the case $d \geq 3$ ? When $d \geq 3$, from [12], for all $\varepsilon>0$, there exists a translation-equivariant matching rule with exponential tails of order $\exp \left(-c r^{d-2-\varepsilon}\right)$, where $c>0$ is some constant. Does there exist a translation-equivariant matching rule with tails of order $\exp \left(-c r^{d}\right)$ ? The original problem is from [8], which also contains a few other related open problems.

2. We say that a translation-equivariant matching rule is oriented if it satisfies the additional restriction that if a site $x$ is matched to a site $y$ that contains a 1 , then $y_{i} \geq x_{i}$ for all $i \leq d$. Observe that in Meshalkin's matching, a 0 is always matched to a 1 that is to the right of it. Note that it is not obvious that the method employed in this paper can be modified to work in an oriented setting. In one dimension, the restriction of orientation does not make a difference; one might think it should not for higher dimensions as well. What is the optimal tail behavior for matchings in $\mathbb{Z}^{d}$ with the restriction that we consider orientation as well?

\section{Acknowledgements}

This research was funded in part by the NSERC and a U.B.C. Graduate Fellowship. I would like to thank my supervisor Ander Holroyd for introducing me to this topic. Our numerous discussions and meetings were invaluable and his assistance and advice have helped to shape all aspects of this work. I would also like to take the opportunity to thank Vlada Limic for introducing me to probability.

\section{References}

[1] Benjamini, I., Lyons, R., Peres, Y. and Schramm, O. (1999). Group-invariant percolation on graphs. Geom. Funct. Anal. 9, 29-66.

[2] Ferrari, P. A., Landim, C. And Thorisson, H. (2004). Poisson trees, succession lines and coalescing random walks. Ann. Inst. H. Poincaré Prob. Statist. 40, 141-152.

[3] HäGgström, O. (1997). Infinite clusters in dependent automorphism invariant percolation on trees. Ann. Prob. 25, 1423-1436.

[4] Hoffman, C., Holroyd, A. E. and Peres, Y. (2006). A stable marriage of Poisson and Lebesgue. Ann. Prob. 34, 1241-1272.

[5] Hoffman, C., Holroyd, A. E. and Peres, Y. (2009). Tail bounds for the stable marriage of Poisson and Lebesgue. Canad. J. Math. 61, 1279-1299.

[6] Holroyd, A. E. AND Liggett, T. M. (2001). How to find an extra head: optimal random shifts of Bernoulli and Poisson random fields. Ann. Prob. 29, 1405-1425.

[7] Holroyd, A. E. And Peres, Y. (2003). Trees and matchings from point processes. Electron. Commun. Prob. 8, $17-27$.

[8] Holroyd, A. E. and Peres, Y. (2005). Extra heads and invariant allocations. Ann. Prob. 33, 31-52.

[9] Holroyd, A. E., Pemantle, R., Peres, Y. and Schramn, O. (2009). Poisson matching. Ann. Inst. H. Poincaré Prob. Statist. 45, 266-287.

[10] Meshalkin, L. (1959). A case of isomorphism of Bernouli schemes. Dokl. Akad. Nauk. SSSR 128, 41-44.

[11] Nazarov, F., Sodin, M. ANd Volberg, A. (2007). Transportation to random zeroes by the gradient flow. Geom. Funct. Anal. 17, 887-935.

[12] Timár, A. (2009). Invariant matchings of exponential tail on coin flips in $\mathbb{Z}^{d}$. Preprint. Available at http://arxiv.org/abs/0909.1009v1.

[13] Timár, A. (2004). Trees and grid factors for general point processes. Electron. Commun. Prob. 9, 53-59. 